COMMUNICATIONS IN

ANALYSIS AND GEOMETRY

Volume 9, Number 2, 239-260, 2001

\title{
Groups quasi-isometric to symmetric spaces
}

\author{
Bruce Kleiner ${ }^{1}$ and Bernhard LeeB ${ }^{2}$
}

\begin{abstract}
We determine the structure of finitely generated groups which are quasi-isometric to nonpositively curved symmetric spaces, allowing Euclidean de Rham factors. If $X$ is a symmetric space of noncompact type (i.e. it has no Euclidean de Rham factor), and $\Gamma$ is a finitely generated group quasi-isometric to the product $\mathbb{E}^{k} \times X$, then there is an exact sequence $1 \rightarrow H \rightarrow \Gamma \rightarrow L \rightarrow 1$ where $H$ contains a finite index copy of $\mathbb{Z}^{k}$ and $L$ is a uniform lattice in the isometry group of $X$.
\end{abstract}

\section{Introduction.}

If $X$ is a symmetric space with no Euclidean de Rham factor, then any finitely generated group $\Gamma$ quasi-isometric to $X$ is a finite extension of a uniform lattice in $\operatorname{Isom}(X)$. This result is a direct corollary of the main results of [KlLe97b] together with earlier work in the rank 1 cases [Tuk88, Gro81a, Hin90, Pan89, Ga92, CJ94], and was first announced in June 1994 at MSRI, and in [KlLe97a]. This result does not extend to symmetric spaces with a nontrivial Euclidean factor: it was observed by Epstein, Gersten, and Mess that any extension of a Fuchsian group by $\mathbb{Z}$ is quasi-isometric to $\mathbb{H}^{2} \times \mathbb{R}$, and such extensions are typically not finite extensions of lattices in Isom $\left(\mathbb{H}^{2} \times \mathbb{R}\right)$. In this paper we treat the case of groups quasi-isometric to symmetric spaces with a Euclidean de Rham factor.

Theorem 1.1. Let $X$ be a symmetric space of noncompact type, and let $N i l$ be a simply connected nilpotent Lie group equipped with a left-invariant Riemannian metric. Suppose $\Gamma$ is a finitely generated group quasi-isometric to $N i l \times X$ (endowed with the product metric). Then there is an exact sequence

$$
1 \longrightarrow H \longrightarrow \Gamma \stackrel{p}{\longrightarrow} L \longrightarrow 1
$$

\footnotetext{
${ }^{1}$ Supported by a Sloan foundation fellowship, and NSF grants DMS-95-05175 and DMS-96-26911.

${ }^{2}$ Supported by SFB 256 (Bonn).
} 
where $H$ is a finitely generated group quasi-isometric to Nil and $L$ is a uniform lattice in the isometry group of $X$, and this sequence is unique up to isomorphism. Furthermore, given any quasi-isometry $\Gamma \stackrel{\phi}{\longrightarrow} N i l \times X$, there is a quasi-isometry $L \stackrel{\bar{\phi}}{\rightarrow} X$ so that the diagram

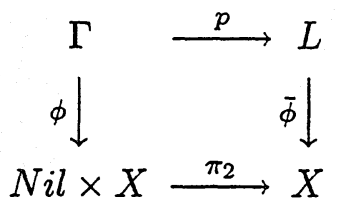

commutes up to bounded error. In particular, $H$ is undistorted ${ }^{3}$ in $\Gamma$.

When $N i l$ is the trivial group then $\Gamma$ is a finite extension of a uniform lattice in $I \operatorname{som}(X)$, and when $N i l \simeq \mathbb{R}^{k}$ then $H$ is virtually abelian of rank $k$ by [Gro81b, Pan83]. The case when $X$ is the hyperbolic plane and $N i l \simeq \mathbb{R}$ is due to Rieffel [Rie93].

We further refine Theorem 1.1 when $N i l \simeq \mathbb{R}^{n}$.

Theorem 1.4. Let $X$ be as in Theorem 1.1. Then any finitely generated group $\Gamma$ quasi-isometric to $\mathbb{R}^{n} \times X$ contains a finite index subgroup $\Gamma_{1} \subset \Gamma$ which is a central extension of the form

$$
1 \longrightarrow \mathbb{Z}^{n} \longrightarrow \Gamma_{1} \longrightarrow L_{1} \longrightarrow 1
$$

where $L_{1}$ is a finite extension of a lattice in $\operatorname{Isom}(X)$.

In general, one cannot arrange that the group $L_{1}$ is a lattice in $\operatorname{Isom}(X)$ rather than a finite extension of a lattice. Examples of Raghunathan [Rag84] show that this is impossible in general even when $n=0$.

Theorem 1.4 raises the question of which central extensions (1.5) are quasi-isometric to $\mathbb{E}^{n} \times X$. Theorem 1.8 below gives a homological answer to this.

Definition 1.6. An extension $1 \rightarrow K \rightarrow G \stackrel{p}{\rightarrow} Q \rightarrow 1$ of finitely generated groups is quasi-isometrically trivial if there is a quasi-isometry $G \stackrel{\phi}{\rightarrow} K \times Q$ so that the diagram

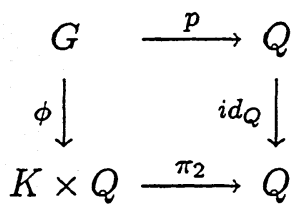

\footnotetext{
${ }^{3}$ The inclusion of $H$ in $\Gamma$ is bilipschitz with respect to the word metrics.
} 
commutes up to bounded error.

The central extension (1.5) is quasi-isometrically trivial by the second part of Theorem 1.1. The next result gives a general characterisation of quasi-isometrically trivial extensions.

Theorem 1.8. (See section 7 for the definition of $L^{\infty}$ cochains for $C W$ complexes.) Let

$$
1 \rightarrow \mathbb{Z}^{n} \rightarrow G \rightarrow Q \rightarrow 1
$$

be a central extension of finitely generated groups, and let $\alpha \in H^{2}\left(Q ; \mathbb{Z}^{n}\right)$ be the associated cohomology class. Let $K$ be a $C W$-complex with finite 1skeleton which is an Eilenberg-Maclane space for $Q$, and identify $\alpha$ with a class in $H^{2}\left(K ; \mathbb{Z}^{n}\right) \simeq H^{2}\left(Q ; \mathbb{Z}^{n}\right)$. Then the extension (1.9) is quasiisometrically trivial iff the pullback of $\alpha$ to $H^{2}\left(\tilde{K} ; \mathbb{Z}^{n}\right)$ is in the image of $H_{L^{\infty}}^{2}\left(\tilde{K} ; \mathbb{Z}^{n}\right) \stackrel{\delta}{\rightarrow} H^{2}\left(\tilde{K}, \mathbb{Z}^{n}\right)$, where $\tilde{K}$ denotes the universal cover of $K$.

Remarks. Using bounded cohomology instead of $L^{\infty}$ cohomology, Gersten [Ger92] gave a sufficient condition for a central extension by $\mathbb{Z}$ to be quasiisometric to a trivial extension. In [ReNe97, Section 4] the authors give another cohomological characterization of quasi-isometrically trivial central extensions.

An earlier version of this paper was posted on the AMS preprint server in October 1996.

We gratefully acknowledge support by the RiP-program at the Mathematisches Forschungsinstitut Oberwolfach.

1 Introduction . . . . . . . . . . . . . . . 239

2 Preliminaries . . . . . . . . . . . . . . . . 242

3 Projecting quasi-actions to the factors . . . . . . . . . 243

4 Straightening cocompact quasi-actions on irreducible symmetric spaces . . . . . . . . . . . . . . . . . . . 244

5 A Growth estimate for small elements in nondiscrete cocompact subgroups of $I s o m(X) \ldots \ldots$. . . . . . 246

5.1 Parabolic isometries of symmetric spaces . . . . . . 246

5.2 The growth estimate . . . . . . . . . . . . 247

6 Proof Theorem $1.1 \ldots \ldots \ldots 250$

7 Proof of Theorem 1.4 . . . . . . . . . . . . . 251

8 Geometry of central extensions by $\mathbb{Z}^{n} \ldots \ldots \ldots 253$

Bibliography . . . . . . . . . . . . . 258 


\section{Preliminaries.}

In this section we recall some basic definitions and notation. See [Gro93] for more discussion and background.

Definition 2.1. A map $f: X \rightarrow Y$ between metric spaces is an $(L, A)$ quasi-isometry if for every $x_{1}, x_{2} \in X$

$$
L^{-1} d\left(x_{1}, x_{2}\right)+A \leq d\left(x_{1}, x_{2}\right) \leq L d\left(x_{1}, x_{2}\right)+A,
$$

and for every $y \in Y$ we have $d(y, f(X))<A$. Two quasi-isometries $f_{1}, f_{2}$ : $X \longrightarrow Y$ are equivalent if $d\left(f_{1}, f_{2}\right)<\infty$.

If $\Gamma$ is a finitely generated group, then any two word metrics on $\Gamma$ are biLipschitz to one another by $i d_{\Gamma}: \Gamma \rightarrow \Gamma$. We will implicitly endow our finitely generated groups with word metrics.

Definition 2.2. An $(L, A)$-quasi-action of a group $\Gamma$ on a metric space $Z$ is a map $\rho: \Gamma \times Z \rightarrow Z$ so that $\rho(\gamma, \cdot): Z \rightarrow Z$ is an $(L, A)$ quasi-isometry for every $\gamma \in \Gamma, d\left(\rho\left(\gamma_{1}, \rho\left(\gamma_{2}, z\right)\right), \rho\left(\gamma_{1} \gamma_{2}, z\right)\right)<A$ for every $\gamma_{1}, \gamma_{2} \in \Gamma, z \in Z$, and $d(\rho(e, z), z)<A$ for every $z \in Z$.

We will denote the self-map $\rho(\gamma, \cdot): Z \rightarrow Z$ by $\rho(\gamma) . \rho$ is discrete if for any point $z \in Z$ and any radius $R>0$, the set of all $\gamma \in \Gamma$ such that $\rho(\gamma, z)$ is contained in the ball $B_{R}(z)$ is finite. $\rho$ is cobounded if $Z$ coincides with a finite tubular neighborhood of the "orbit" $\rho(\Gamma) z \subset Z$ for every $z$. If $\rho$ is a discrete cobounded quasi-action of a finitely generated group $\Gamma$ on a geodesic metric space $Z$, it follows easily that the map $\Gamma \rightarrow Z$ given by $\gamma \mapsto \rho(\gamma, z)$ is a quasi-isometry for every $z \in Z$.

Definition 2.3. Two quasi-actions $\rho$ and $\rho^{\prime}$ are equivalent if there exists a constant $D$ so that $d\left(\rho(\gamma), \rho^{\prime}(\gamma)\right)<D$ for all $\gamma \in \Gamma$.

Definition 2.4. Let $\rho$ and $\rho^{\prime}$ be a quasi-actions of $\Gamma$ on $Z$ and $Z^{\prime}$ respectively, and let $\phi: Z \rightarrow Z^{\prime}$ be a quasi-isometry. Then $\rho$ is quasi-isometrically conjugate to $\rho^{\prime}$ via $\phi$ if there is a $D$ so that $d\left(\phi \circ \rho(\gamma), \rho^{\prime}(\gamma) \circ \phi\right)<D$ for all $\gamma \in \Gamma$.

Lemma 2.5 (cf. [Gro87, 8.2.K]). Let $X$ be a Hadamard manifold of dimension $\geq 2$ with sectional curvature $\leq K<0$, and let $\partial_{\infty} X$ denote the geometric boundary of $X$ with the cone topology. Recall that every quasiisometry $\Phi: X \longrightarrow X$ induces a boundary homeomorphism $\partial_{\infty} \Phi: \partial_{\infty} X \rightarrow$ $\partial_{\infty} X$. 
1. If $\rho: \Gamma \times X \rightarrow X$ is a quasi-action on $X$, then $\rho$ is discrete (respectively cobounded) iff $\partial_{\infty} \phi$ acts properly discontinuously (respectively cocompactly) on the space of distinct triples in $\partial_{\infty} X$.

2. Given $(L, A)$ there is a $D$ so that if $\phi_{k}, \psi$ are $(L, A)$ quasi-isometries, then $\partial_{\infty} \phi_{k}$ converges uniformly to $\partial_{\infty} \psi$ iff $\lim \sup d\left(\phi_{k} x, \psi x\right)<D$ for every $x \in X$. In particular, if $\phi_{1}, \phi_{2}: X \longrightarrow X$ are $(L, A)$ quasiisometries with the same boundary mappings, then $d\left(\phi_{1}, \phi_{2}\right)<D$.

Proof. Let $\partial^{3} X \subset \partial_{\infty} X \times \partial_{\infty} X \times \partial_{\infty} X$ denote the subspace of distinct triples. The uniform negative curvature of $X$ implies that there is a $D_{0}$ depending only on $K$ such that

(a) For every $x \in X$ there is a triple $\left(\xi_{1}, \xi_{2}, \xi_{3}\right) \in \partial^{3} X$ such that $d\left(x, \overline{\xi_{i} \xi_{j}}\right)<D_{0}$ for every $1 \leq i \neq j \leq 3$, where $\overline{\xi_{i} \xi_{j}}$ denotes the geodesic with ideal endpoints $\xi_{i}, \xi_{j}$. Moreover for every $C$ the set $\left\{\left(\xi_{1}, \xi_{2}, \xi_{3}\right) \mid d\left(x, \overline{\xi_{i} \xi_{j}}\right)<C\right.$ for all $\left.1 \leq i \neq j \leq 3\right\}$ has compact closure in $\partial^{3} X$.

and

(b) For every $\left(\xi_{1}, \xi_{2}, \xi_{3}\right) \in \partial^{3} X$ there is a point $x \in X$ so that $d\left(x, \overline{\xi_{i} \xi_{j}}\right)<D_{0}$ for each $1 \leq i \neq j \leq 3$. And for every $C$ there is a $C^{\prime}$ depending only on $C$ and $K$ so that $\{x \in X$ । $d\left(x, \overline{\xi_{i} \xi_{j}}\right)<C$ for every $\left.1 \leq i \neq j \leq 3\right\}$ has diameter $<C^{\prime}$.

easily from this.

\section{Projecting quasi-actions to the factors.}

Let $N i l$ and $X$ be as in Theorem 1.1 and decompose $X$ into irreducible factors:

$$
X=\prod_{i=1}^{l} X_{i}
$$

Suppose $\rho$ is a quasi-action of the finitely generated group $\Gamma$ on $N i l \times X$. We denote by $p: N i l \times X \rightarrow X$ the canonical projection. Theorem 1.1.2 from $[\mathrm{KlLe} 97 \mathrm{~b}]^{4}$ implies that every quasi-isometry of $N i l \times X$ respects the

\footnotetext{
${ }^{4}$ Although Theorem 1.1.2 is only formulated in the case that $N i l \simeq \mathbb{R}^{n}$, the same proof works in general provided one uses [Pan83] to conclude that all asymptotic cones of $N i l$ are homeomorphic to $\mathbb{R}^{k}$ where $k=\operatorname{Dim}(N i l)$.
} 
fibering $p$ and covers a product quasi-isometry $X \rightarrow X$, up to bounded error. Applying this theorem to $\rho(\gamma)$ for each $\gamma$, we construct quasi-actions $\rho_{i}$ of $\Gamma$ on $X_{i}$ so that

$$
d\left(p \circ \rho(\gamma), \prod_{i=1}^{k} \rho_{i}(\gamma) \circ p\right)<D
$$

for all $\gamma \in \Gamma$ and some positive constant $D$.

\section{Straightening cocompact quasi-actions on irreducible symmetric spaces.}

The following result is a direct consequence of [Pan89, Théorème 1] and [KlLe97b, Theorem 1.1.3].

Fact 4.1. Let $X$ be an irreducible symmetric space other than a real or complex hyperbolic space. Then every quasi-action on $X$ is equivalent to an isometric action.

Proof. Let $\rho$ be a quasi-action of a group $\Gamma$ on $X$. By the results just cited, there is an isometry $\bar{\rho}(\gamma)$ at finite distance from the quasi-isometry $\rho(\gamma)$ for every $\gamma \in \Gamma$. This isometry is unique and its distance from $\rho(\gamma)$ is uniformly bounded ${ }^{5}$ in terms of the constants of the quasi-action. So $\bar{\rho}$ is an isometric action equivalent to $\rho$.

We recall that the real and complex hyperbolic spaces of all dimensions admit quasi-isometries which are not equivalent to isometries [Pan89].

Fact 4.2. Any cobounded quasi-action $\rho$ on a real or complex hyperbolic space of dimension $>2$ is quasi-isometrically conjugate to an isometric action.

This result is due to Sullivan in the $\mathbb{H}^{3}$ case, and to [Gro81a, Tuk86] in the real-hyperbolic case. Using Pansu's theory of Carnot differentiability one can carry out Tukia's arguments for all rank-one symmetric spaces other than hyperbolic plane, cf. [Pan89, sec. 11]. Another proof for the complexhyperbolic case can be found in [Chow96].

\footnotetext{
${ }^{5}$ The uniformity in the rank one case follows from Lemma 2.5 .
} 
Fact 4.3. Let $\rho$ be a cobounded quasi-action of a group $\Gamma$ on $\mathbb{H}^{2}$. Then $\rho$ is quasi-isometrically conjugate to a cocompact isometric action of $\Gamma$ on $\mathbb{H}^{2}$.

Proof. We recall that every quasi-isometry $\phi: \mathbb{H}^{2} \rightarrow \mathbb{H}^{2}$ induces a quasisymmetric homeomorphism $\partial_{\infty} \phi: \partial_{\infty} \mathbb{H}^{2} \rightarrow \partial_{\infty} \mathbb{H}^{2}$, see [TuVa82]; moreover the quasi-symmetry constant of $\partial_{\infty} \phi$ can be estimated in terms of the quasiisometry constants of $\phi$. Since equivalent quasi-isometries yield the same boundary homeomorphism, every quasi-action $\rho$ on $\mathbb{H}^{2}$ induces a genuine action $\partial_{\infty} \rho$ on $\partial_{\infty} \mathbb{H}^{2}$ by uniformly quasi-symmetric homeomorphisms.

Let $\bar{\Gamma}$ be the quotient of $\Gamma$ by the kernel of the action $\partial_{\infty} \rho$, and let $\pi: \Gamma \rightarrow \bar{\Gamma}$ be the canonical epimorphism. If two elements $\gamma_{1}, \gamma_{2} \in \Gamma$ have the same boundary map then $d\left(\rho\left(\gamma_{1}\right), \rho\left(\gamma_{2}\right)\right)$ is uniformly bounded by Lemma 2.5. Hence we may obtain a quasi-action $\bar{\rho}$ of $\bar{\Gamma}$ on $\mathbb{H}^{2}$ by choosing $\gamma \in \pi^{-1}(\bar{\gamma})$ for each $\bar{\gamma} \in \bar{\Gamma}$, and setting $\bar{\rho}(\bar{\gamma})=\rho(\gamma)$. If $\bar{\tau}$ is an isometric action of $\bar{\Gamma}$ on $\mathbb{H}^{2}$ and $\phi: \mathbb{H}^{2} \rightarrow \mathbb{H}^{2}$ quasi-isometrically conjugates $\bar{\rho}$ into $\bar{\tau}$, then $\phi$ will quasi-isometrically conjugate $\rho$ into the isometric action $\tau: \Gamma \times \mathbb{H}^{2} \rightarrow \mathbb{H}^{2}$ given by $\tau(\gamma)=\bar{\tau}(\pi(\gamma))$. Hence it suffices to treat the case when $\bar{\Gamma}=\Gamma$, and so we will assume that $\partial_{\infty} \rho$ is an effective action.

Lemma 4.4. The quasi-action $\rho$ is discrete if and only if the action $\partial_{\infty} \rho$ on $\partial_{\infty} \mathbb{H}^{2}$ is discrete in the compact-open topology.

Proof. Suppose $\partial_{\infty} \rho$ is discrete, and let $\left(\gamma_{i}\right)$ be a sequence in $\Gamma$ so that $\rho\left(\gamma_{i}\right)$ maps a point $p \in \mathbb{H}^{2}$ into a fixed ball $B_{R}(p)$. Then by a selection argument we may assume - after passing to a subsequence if necessary that there is a quasi-isometry $\phi: \mathbb{H}^{2} \rightarrow \mathbb{H}^{2}$ so that for every $q \in \mathbb{H}^{2}$ we have $\limsup _{i} d\left(\rho\left(\gamma_{i}\right)(q), \phi(q)\right)<D$ for some $D$. Hence the boundary maps $\partial_{\infty} \rho\left(\gamma_{i}\right)$ converge to $\partial_{\infty} \phi$, and so the sequence $\partial_{\infty} \rho\left(\gamma_{i}\right)$ is eventually constant. Since $\rho$ is effective we conclude that $\gamma_{i}$ is eventually constant. Therefore $\rho$ is a discrete quasi-action.

If $\rho$ is a discrete quasi-action on $\mathbb{H}^{2}$, then $\partial_{\infty} \rho$ is discrete by Lemma 2.5 .

Proof of 4.3 continued.

Case 1: $\partial_{\infty} \rho$ is discrete. In this case, $\rho$ is a discrete convergence group action (Lemma 2.5) and by the work of [CJ94, Ga92], there is a discrete isometric action $\tau$ of $\Gamma$ on $\mathbb{H}^{2}$ so that $\partial_{\infty} \rho$ is topologically conjugate to $\partial_{\infty} \tau$. 
Since $\rho$ is cobounded, $\partial_{\infty} \rho$ acts cocompactly on the set of distinct triples of points in $\partial_{\infty} \mathbb{H}^{2}$ (lemma 2.5); therefore $\partial_{\infty} \tau$ also acts cocompactly on the space of triples and so $\tau$ is a discrete, cocompact, isometric action of $\Gamma$ on $\mathbb{H}^{2}$. We now have two discrete, cobounded, quasi-actions of $\Gamma$ on $\mathbb{H}^{2}$, so they are quasi-isometrically conjugate by some quasi-isometry $\psi: \mathbb{H}^{2} \rightarrow \mathbb{H}^{2}$.

Case 2: $\partial_{\infty} \rho$ is nondiscrete. By [Hin90, Theorem 4], $\partial_{\infty} \rho$ is quasisymmetrically conjugate to $\partial_{\infty} \tau$, where $\tau$ is an isometric action on $\mathbb{H}^{2}$. The conjugating quasi-symmetric homeomorphism is the boundary of a quasiisometry $\psi: \mathbb{H}^{2} \rightarrow \mathbb{H}^{2}$, [TuVa82], which quasi-isometrically conjugates $\partial_{\infty} \rho$ into the isometric action action $\tau$. Applying Lemma 2.5 again, we conclude that $\tau$ is cocompact.

Section 3, and facts $4.1,4.2$ and 4.3 imply:

Corollary 4.5. Let $X$ be a symmetric space of noncompact type without Euclidean factor. Then any cobounded quasi-action on $X$ is quasi-isometrically conjugate to a cocompact isometric action on $X$.

\section{A Growth estimate for small elements in nondiscrete cocompact subgroups of $I \operatorname{som}(X)$.}

\subsection{Parabolic isometries of symmetric spaces.}

Let $X$ be a symmetric space of noncompact type, and let $G=I \operatorname{som}(X)$.

Recall that the displacement function of an isometry $g$ is the convex function $\delta_{g}: X \rightarrow \mathbb{R}$ defined by the formula $\delta_{g}(x):=d(g x, x)$. An isometry $g \in G$ is semisimple if its displacement function $\delta_{g}$ attains its infimum and parabolic otherwise.

Lemma 5.1. Let $A \subset G$ be a finitely generated abelian group all of whose nontrivial elements are parabolic. Then $A$ has a fixed point at infinity.

Proof. Recall that the nearest point projection to a closed convex subset is well-defined and distance non-increasing. This implies that if $C$ is a nonempty $A$-invariant closed convex set, then for all displacement functions $\delta_{a}$, $a \in A$, we have $\inf \delta_{a}=\left.\inf \delta_{a}\right|_{C}$. Hence for all $n \in \mathbb{N}$, the intersection of the sublevel sets $\left\{p \mid \delta_{a_{i}}(p) \leq \inf \delta_{a_{i}}+1 / n\right\}$ is non-empty and contains a point $p_{n}$. We have $\delta_{a_{i}}\left(p_{n}\right) \rightarrow \inf \delta_{a_{i}}$ for all $a_{i}$, and since the isometries $a_{i}$ 
are parabolic the sequence $\left\{p_{n}\right\}$ subconverges to an ideal boundary point $\xi \in \partial_{\infty} X$. It follows that the $a_{i}$ fix $\xi$.

Lemma 5.2. Let $a_{1}, \ldots, a_{k} \in I$ som $(X)$ be commuting parabolic isometries. Then there is a sequence of isometries $\left\{g_{n}\right\} \subset G$ so that for every $i$ the sequence $g_{n} a_{i} g_{n}^{-1}$ subconverges to a semisimple isometry $\bar{a}_{i}$.

Proof. From the proof of the previous lemma, there is a sequence of points $\left\{p_{n}\right\} \subset X$ converging to an ideal point $\xi$ so that $\delta_{a_{i}}\left(p_{n}\right) \rightarrow \inf \delta_{a_{i}}$ for all $a_{i}$. Pick isometries $g_{n} \in G$ such that $g_{n} \cdot p_{n}=p_{0}$. The conjugates $g_{n} a_{i} g_{n}^{-1}$ have the same infimum displacement as $a_{i}$. Since

$$
\delta_{g_{n} a_{i} g_{n}^{-1}}\left(p_{0}\right)=\delta_{a_{i}}\left(p_{n}\right) \rightarrow \inf \delta_{a_{i}},
$$

the $g_{n} a_{i} g_{n}^{-1}$ subconverge to a semisimple isometry.

We call an isometry $g \neq e$ purely parabolic ${ }^{6}$ if the identity is the only semisimple element in $\overline{A d_{G}(G) \cdot g}$.

\subsection{The growth estimate.}

Proposition 5.3. Let $X$ be a symmetric space of noncompact type with no Euclidean de Rham factors. Let $\Gamma \subset G=I \operatorname{som}(X)$ be a finitely generated, nondiscrete, cocompact subgroup. Let $U \subset \operatorname{Isom}(X)$ be a neighborhood of the identity, and set

$$
f(k):=\#\left\{g \in \Gamma:|g|_{\Gamma}<k, g \in U\right\},
$$

where $|\cdot| \Gamma$ denotes a word norm on $\Gamma$. Then $f$ grows faster than any polynomial, i.e. for every $d>0 \lim \sup _{k \rightarrow \infty} \frac{f(k)}{k^{d}}=\infty$.

Proof. Let $\bar{\Gamma}$ be the closure of $\Gamma$ in $G$ with respect to the Hausdorff topology, and let $\bar{\Gamma}^{\circ}$ be the identity component of $\bar{\Gamma}$.

Case 1: $\bar{\Gamma}^{\circ}$ is nilpotent. Let $A$ be the last non-trivial subgroup in the derived series of $\bar{\Gamma}^{o}$. Then $A \subset \bar{\Gamma}$ is a connected abelian subgroup of positive dimension, $A$ is normal in $\bar{\Gamma}$, and $\Gamma \cap A$ is dense in $A$.

Lemma 5.4. For every $\delta \in(0,1)$ there is a $\gamma \in \Gamma$ such that all eigenvalues of the automorphism $\left.A d_{G}(\gamma)\right|_{A}: A \rightarrow A$ have absolute value $<\delta$.

\footnotetext{
${ }^{6}$ This is a geometric way of defining unipotent isometries.
} 
Proof. See section 5.1 for terminology.

Step 1: A contains no semisimple isometries other than e. Otherwise we can consider the intersection $C$ of the minimum sets for the displacement functions $\delta_{a}$ where $a$ runs through all semisimple elements in $A . C$ is a nonempty convex subset of $X$ which splits metrically as $C \cong \mathbb{E}^{k} \times Y$. The flats $\mathbb{E}^{k} \times\{y\}$ are the minimal flats preserved by all semisimple elements in $A$. Since $\Gamma$ normalises $A$ it follows that $C$ is $\Gamma$-invariant. The cocompactness of $\Gamma$ implies that $C=X$ and $k=0$ because $X$ has no Euclidean factor. This means that the semisimple elements in $A$ fix all points, a contradiction.

Step 2: All non-trivial isometries in $A$ are purely parabolic. If $a \in A$, $a \neq e$, is not purely parabolic then there is a sequence of isometries $g_{n}$ so that $g_{n} a g_{n}^{-1}$ converges to a semisimple isometry $\bar{a} \neq e$. We can uniformly approximate the $g_{n}$ by elements in $\Gamma$, i.e. there exist $\gamma_{n} \in \Gamma$ and a bounded sequence $k_{n} \in G$ subconverging to $k \in G$ so that $\gamma_{n}=k_{n} g_{n}$. Then $\gamma_{n} a \gamma_{n}^{-1}=k_{n} g_{n} a g_{n}^{-1} k_{n}^{-1}$ subconverges to the non-trivial semisimple element $k \bar{a} k^{-1}$. This contradicts step 1 .

Step 3: Pick a basis $\left\{a_{1}, \ldots, a_{k}\right\}$ for $A \simeq \mathbb{R}^{k}$. By Lemma 5.2 there exist elements $g_{n} \in G$ so that $g_{n} a_{i} g_{n}^{-1} \rightarrow e$ for all $a_{i}$. We approximate the $g_{n}$ as above by $\gamma_{n}$ so that the sequence $\gamma_{n} g_{n}^{-1}$ is bounded. Then $\gamma_{n} a_{i} \gamma_{n}^{-1} \rightarrow e$ for all $a_{i}$. The lemma follows by setting $\gamma=\gamma_{n}$ for sufficiently large $n$.

Proof of case 1 continued. By Lemma 5.4, there is a $\gamma \in \Gamma, \gamma \neq e$, and a norm $\|\cdot\|_{A}$ on $A$ such that for all $a \in A$ we have

$$
\left\|\gamma a \gamma^{-1}\right\|_{A}<\frac{1}{2}\|a\|_{A} .
$$

Consider a neighborhood $U$ of $e$ in $G$. Let $r>0$ be small enough so that $\left\{a \in A:\|a\|_{A}<r\right\} \subset U$ and pick $\alpha \in \Gamma \cap A$ with $\|\alpha\|_{A}<r / 2$. Then the elements

$$
\gamma_{\epsilon_{0} \ldots \epsilon_{n-1}}=\alpha^{\epsilon_{0}} \cdot\left(\gamma \alpha \gamma^{-1}\right)^{\epsilon_{1}} \cdots\left(\gamma^{n-1} \alpha \gamma^{1-n}\right)^{\epsilon_{n-1}}
$$

for $\epsilon_{i} \in\{0,1\}$ are $2^{n}$ pairwise distinct elements contained in $\Gamma \cap U$ with word

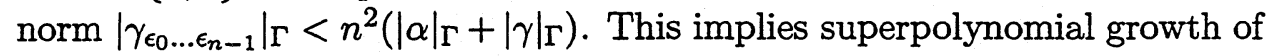
$f$.

Case 2: $\bar{\Gamma}^{\circ}$ is not nilpotent. Define an increasing sequence (the upper central series) of nilpotent Lie subgroups $Z_{i} \subset \bar{\Gamma}^{\circ}$ inductively as follows: Set $Z_{0}=$ $\{e\}$ and let $Z_{i+1}$ be the inverse image in $\bar{\Gamma}^{\circ}$ of the center in $\bar{\Gamma}^{o} / Z_{i}$. The dimension of $Z_{i}$ stabilizes and we choose $k$ so that $\operatorname{dim} Z_{k}$ is maximal. Then 
the center of $\bar{\Gamma} / Z_{k}$ is discrete and, since $\bar{\Gamma}^{o}$ is not nilpotent, we have $\operatorname{dim} Z_{k}<$ $\operatorname{dim} \bar{\Gamma}$. Proposition 5.3 now follows by applying the next lemma with $H=\bar{\Gamma}$ and $H_{1}=Z_{k}$.

Lemma 5.5. Let $H$ be a Lie group, let $H_{1} \triangleleft H$ be a closed normal subgroup so that $\bar{H}:=H / H_{1}$ is a positive dimensional Lie group with discrete center, and suppose $\Gamma \subset H$ is a dense, finitely generated subgroup. If $U$ is any neighborhood of $e$ in $H$, then the function $f(k):=\#\left\{g \in \Gamma:|g|_{\Gamma} \leq k, g \in U\right\}$ grows superpolynomially.

Proof. The idea of the proof is to use the contracting property of commutators to produce a sequence $\left\{\alpha_{k}\right\}$ in $H \cap \Gamma$ which converges exponentially to the identity. The word norm $\left|\alpha_{k}\right|_{\Gamma}$ grows exponentially with $k$, but the number of elements of $\left\langle\alpha_{1}, \ldots, \alpha_{k}\right\rangle$ in $U$ also grows exponentially with $k$; by comparing growth exponents we find that $f$ grows superpolynomially.

Fix $M \in \mathbb{N}$, a positive real number $\epsilon<1 / 3$ and some left-invariant Riemannian metric on $H$. Since the differential of the commutator map $\left(h, h^{\prime}\right) \mapsto\left[h, h^{\prime}\right]$ vanishes at $(e, e)$ we can find a neighborhood $V$ of $e$ in $H$ such that:

$$
h, h^{\prime} \in V \quad \Longrightarrow \quad\left[h, h^{\prime}\right] \in V \quad \text { and } \quad d\left(\left[h, h^{\prime}\right], e\right)<\frac{1}{4 M} d(h, e)
$$

Since the differential of the $k$-th power $h \mapsto h^{k}$ at $e$ is $k \cdot i d_{T_{e} H}$ for all $k \in \mathbb{Z}$, we can furthermore achieve that, whenever $1 \leq k, k^{\prime} \leq M^{\prime}$ and $h, h^{k}, h^{k^{\prime}} \in V$, then

$$
d\left(h^{k}, h^{k^{\prime}}\right) \geq\left(\left|k-k^{\prime}\right|-\epsilon\right) \cdot d(h, e)
$$

By our assumption, there exist finitely many elements $\gamma_{1}, \ldots, \gamma_{m} \in \Gamma \cap$ $V$ such that the centralizers $Z_{\bar{H}}\left(\bar{\gamma}_{j}\right)$ of their images in $\bar{H}$ have discrete intersubsection. We construct an infinite sequence of elements $\alpha_{i} \in(\Gamma \cap$ $V) \backslash H_{1}$ by picking $\alpha_{0} \in V$ arbitrarily and setting $\alpha_{i+1}=\left[\alpha_{i}, \gamma_{j(i)}\right] \notin H_{1}$ for suitably chosen $1 \leq j(i) \leq m$. Then

$$
0<d\left(\alpha_{i+1}, e\right)<\frac{1}{4 M} d\left(\alpha_{i}, e\right)
$$

by (5.6).

Sublemma 5.9. Pick $n_{0} \in \mathbb{N}$. The $M^{n}$ elements

$$
\gamma_{\epsilon_{1} \ldots \epsilon_{n}}=\alpha_{n_{0}+1}^{\epsilon_{1}} \cdots \alpha_{n_{0}+n}^{\epsilon_{n}} \quad \epsilon_{i} \in\{0, \ldots, M-1\}
$$

are distinct. 
Proof. Assume that $\gamma_{\epsilon_{1} \ldots \epsilon_{n}}=\gamma_{\epsilon_{1}^{\prime} \ldots \epsilon_{n}^{\prime}}, \epsilon_{l} \neq \epsilon_{l}^{\prime}$ and $\epsilon_{i}=\epsilon_{i}^{\prime}$ for all $i<l$. Then

$$
\alpha_{n_{0}+l}^{\epsilon_{l}-\epsilon_{l}^{\prime}}=\left(\alpha_{n_{0}+l+1}^{\epsilon_{l+1}^{\prime}} \ldots \alpha_{n_{0}+n}^{\epsilon_{n}^{\prime}}\right)\left(\alpha_{n_{0}+l+1}^{\epsilon_{l+1}} \ldots \alpha_{n_{0}+n}^{\epsilon_{n}}\right)^{-1} .
$$

By (5.8) and the triangle inequality

$$
\begin{aligned}
d\left(\left(\alpha_{n_{0}+l+1}^{\epsilon_{l+1}^{\prime}} \ldots \alpha_{n_{0}+n}^{\epsilon_{n}^{\prime}}\right)\left(\alpha_{n_{0}+l+1}^{\epsilon_{l+1}} \ldots \alpha_{n_{0}+n}^{\epsilon_{n}}\right)^{-1}, e\right) \\
\quad<2 M \sum_{j=1}^{\infty} \frac{1}{(4 M)^{j}} d\left(\alpha_{n_{0}+l}, e\right) \leq \frac{2}{3} d\left(\alpha_{n_{0}+l}, e\right)
\end{aligned}
$$

On the other hand, by (5.7) we have

$$
d\left(\alpha_{n_{0}+l}^{\epsilon_{l}-\epsilon_{l}^{\prime}}, e\right) \geq(1-\epsilon) d\left(\alpha_{n_{0}+l}, e\right)>\frac{2}{3} d\left(\alpha_{n_{0}+l}, e\right)
$$

which is a contradiction.

To complete the proof of the lemma, we observe that the elements (5.10) have word norm $\left|\gamma_{\epsilon_{1} \ldots \epsilon_{n}}\right| \Gamma \leq \operatorname{const}\left(n_{0}\right) \cdot 3^{n}$ and are contained in $U$ if $n_{0}$ is sufficiently large. This shows that $f(k)$ grows polynomially of order at least $\frac{\log (M)}{\log (3)}$ for all $M$, hence the claim.

\section{Proof Theorem 1.1.}

Let $\rho_{0}: \Gamma \times \Gamma \rightarrow \Gamma$ be the isometric action of $\Gamma$ on itself by left translation, and let $\phi: \Gamma \rightarrow N i l \times X$ be a quasi-isometry. Then there is a quasi-action $\rho$ of $\Gamma$ on $N i l \times X$ such that $\phi$ quasi-isometrically conjugates $\rho_{0}$ into $\rho$. According to section $3, \rho$ projects (up to bounded error) to a cobounded quasi-action $\bar{\rho}$ of $\Gamma$ on $X . \bar{\rho}$ is quasi-isometrically conjugate to a cocompact isometric action $\hat{\rho}$, cf. Corollary 4.5. Pick $x \in X, y \in N i l \times\{x\}$, and $R>0$. Since the quasi-action $\rho$ covers $\bar{\rho}$, we know that for all $\gamma \in \Gamma$ with $\hat{\rho}(\gamma) \cdot x \in B_{R}(x)$, the distance $d(\rho(\gamma) \cdot y, N i l \times\{x\})$ is uniformly bounded. The map $\Gamma \rightarrow N i l \times X$ given by $\gamma \mapsto \rho(\gamma) \cdot y$ being a quasi-isometry, we conclude that the function

$$
N(k):=\#\left\{\gamma \in \Gamma|| \gamma \mid \Gamma<k, \hat{\rho}(\gamma) \cdot x \in B_{R}(x)\right\}
$$

grows at most as fast as the volume of balls in $N i l$, i.e. it is $<C k^{d}$ for some $C, d \in \mathbb{R}$. Proposition 5.3 implies that $L:=\hat{\rho}(\Gamma)$ is a discrete subgroup in 
$\operatorname{Isom}(X)$ and hence a uniform lattice. The kernel $H$ of the action $\hat{\rho}$ is then a finitely generated group quasi-isometric to the fiber $N i l$, since it clearly (quasi)-acts discretely and coboundedly on the fiber.

To see that the sequence (1.2) is unique up to isomorphism, let

$$
1 \rightarrow H^{\prime} \rightarrow \Gamma \stackrel{p^{\prime}}{\rightarrow} L^{\prime} \rightarrow 1
$$

be an exact sequence with $L^{\prime} \subset I \operatorname{som}(X)$ a uniform lattice and $H^{\prime}$ a group quasi-isometric to $N i l$. Then by [Gro81b, Pan83] $H^{\prime}$ is a virtually nilpotent group. Now if $\Gamma \stackrel{f}{\rightarrow} \Gamma$ is an isomorphism then $p^{\prime}(H) \subset L^{\prime}$ is a normal, finitely generated, virtually nilpotent subgroup; it follows that $p^{\prime}(f(H))$ is trivial. Similarly $p\left(f^{-1}\left(H^{\prime}\right)\right)$ is trivial and we conclude that $f$ induces an isomorphism of the two exact sequences.

We now prove the last statement of Theorem 1.1. When we restrict $\bar{\rho}$ to $H$ we get a quasi-action which is equivalent to the trivial action of $H$ on $X$. Hence $\bar{\rho}$ induces a quasi-action $\eta$ of $L=\Gamma / H$ on $X$, which is discrete and cobounded. The action $\eta_{0}$ of $L$ on itself by left translations is also discrete and cobounded, so $g \mapsto \eta(g)\left(\pi_{2}(\phi(e))\right)$ defines a quasi-isometry $L \stackrel{\bar{\phi}}{\rightarrow} X$. It follows that the diagram

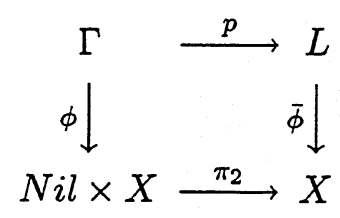

commutes up to bounded error since $\phi$ quasi-isometrically conjugates $\rho_{0}$ into $\rho, \rho$ projects to $\bar{\rho}$, and $d(\bar{\rho}(\gamma H), \eta(\gamma H))$ is uniformly bounded (independent of $\gamma)$.

\section{Proof of Theorem 1.4.}

Sketch of proof. If $\Gamma$ is quasi-isometric to $\mathbb{R}^{n} \times X$ where $X$ is a symmetric space with no Euclidean de Rham factor, then by Theorem 1.1, $\Gamma$ fits into an exact sequence (1.2) where $H$ is an undistorted virtually $\mathbb{Z}^{n}$ subgroup. We will use the undistortedness of $H$ to pass to a finite index subgroup of $\Gamma$ which is a central extension, cf. [Ger91].

If $S$ is a subset of a group $G$, we will use the notation $Z(S, G)$ to denote the centralizer of $S$ in $G$, and $Z(G)$ to denote the center of $G$. 
Proof of Theorem 1.4. By Theorem 1.1 we get an exact sequence

$$
1 \longrightarrow H \longrightarrow \Gamma \stackrel{p}{\longrightarrow} L \longrightarrow 1
$$

where $H$ is a finitely generated group quasi-isometric to $\mathbb{Z}^{n}$, and $L \subset$ $\operatorname{Isom}(X)$ is a uniform lattice. Applying the second part of the theorem we can get a quasi-isometry $\Gamma \stackrel{f}{\longrightarrow} \mathbb{Z}^{n} \times L$ so that

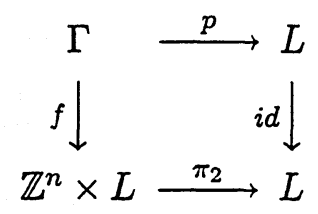

commutes up to bounded error. Clearly $f(H) \subset \mathbb{Z}^{n} \times L$ has finite Hausdorff distance from $\mathbb{Z}^{n} \times\{e\} \subset \mathbb{Z}^{n} \times L$, so $H$ is undistorted ${ }^{7}$ in $\Gamma$. By [Gro81b, Pan83] $H$ contains a finite index copy of $\mathbb{Z}^{n}$.

Next we will identify a finite index abelian subgroup of $H$ which is normal in $\Gamma$. Let $T$ be the subgroup of "translations" in $H$, i.e.

$$
T=\{h \in H \mid[H: Z(h, H)]<\infty\} .
$$

Clearly $T$ is a characteristic subgroup of $H$, and has finite index in $H$; in particular $T$ is finitely generated. Note that $Z(T)$, the center of $T$, has finite index in $T$ since if $T=\left\langle t_{1}, \ldots, t_{k}\right\rangle$, then $Z(T)=\cap_{i} Z\left(t_{i}, T\right)$ is a finite intersection of finite index subgroups of $T$. Hence $Z(T)$ is a finitely generated abelian group of the form $\mathbb{Z}^{n} \oplus A$ where $A$ is a finite abelian group. Note $Z(T)$ is normal in $\Gamma$ since it is characteristic in $H$, and $H$ is normal in $\Gamma$.

Lemma 7.3. The centralizer of $Z(T)$ in $\Gamma, Z(Z(T), \Gamma)$, has finite index in $\Gamma$.

The proof uses properties of translation numbers, see [Gro81a, pp. 189191]. The paper [Ger91] uses a similar setup.

Definition 7.4. Let $G$ be a finitely generated group, and let $|\cdot|_{G}$ be a word norm on $G$. Then the translation length of $g \in G$ is

$$
\delta_{G}(g):=\lim _{k \rightarrow \infty} \frac{\left|g^{k}\right|_{G}}{k} .
$$

The limit exists since $k \mapsto\left|g^{k}\right|_{G}$ is a subadditive function.

\footnotetext{
${ }^{7} \mathrm{~A}$ finitely generated subgroup of a finitely generated group is undistorted if the inclusion homomorphism is a quasi-isometric embedding.
} 
The translation length is conjugacy invariant, vanishes on torsion elements, and changes by at most a bounded factor if one passes to a different word metric. If a homomorphism $\alpha: H \rightarrow G$ is a quasi-isometric embedding of finitely generated groups (i.e. $\exists C>0$ such that $|\alpha(h)|_{G} \geq C|h|_{H}$ for all $h \in H$ ) then the pullback of $\delta_{G}$ to $H$ agrees with $\delta_{H}$ up to a bounded factor.

Proof of Lemma 7.3. We know that $Z(T)$ is undistorted in $\Gamma$ since $Z(T)$ has finite index in $H$ and $H$ is undistorted in $\Gamma$. Hence $\delta_{\Gamma}$ restricts to a function on $Z(T)$ which is equivalent to $\delta_{Z(T)}$. The latter function clearly factors through the homomorphism $Z(T) \rightarrow \mathbb{Z}^{n}$ whose kernel is the torsion subgroup $A \subset Z(T)$. Hence $\delta_{Z(T)}: Z(T) \rightarrow \mathbb{R}$ is a proper function on $Z(T)$ which is invariant under conjugacy by elements of $\Gamma$. If $R$ is large enough that $K_{R}:=\left\{g \in Z(T) \mid \delta_{\Gamma}(g) \leq R\right\}$ generates $Z(T)$, then any finite index subgroup of $\Gamma$ centralizing $K_{R}$ will centralize $Z(T)$, so $Z(Z(T), \Gamma)$ has finite index in $\Gamma$.

Proof of Theorem 1.4 concluded. Let $\Gamma_{1}:=Z(Z(T), \Gamma)$, let $H_{1} \subseteq Z(T) \subseteq$ $\Gamma_{1} \cap H$ be a finite index subgroup of $Z(T)$ isomorphic to $\mathbb{Z}^{n}$, and set $L_{1}:=$ $\Gamma_{1} / H_{1}$. Then clearly $L_{1}$ is a finite extension of a uniform lattice in $I \operatorname{som}(X)$, and hence

$$
1 \rightarrow H_{1} \rightarrow \Gamma_{1} \rightarrow L_{1} \rightarrow 1
$$

is an exact sequence as in (1.5).

\section{Geometry of central extensions by $\mathbb{Z}^{n}$.}

The objective of this section is Proposition 8.3, which provides criteria for recognizing quasi-isometrically trivial central extensions.

Definition 8.1. Let $X$ be a CW-complex. A cellular $k$-cochain $\alpha \in$ $C^{k}\left(X ; \mathbb{Z}^{n}\right)$ is bounded if its values on the $k$-cells of $X$ are uniformly bounded. The collection of bounded cochains forms a subgroup ${ }^{8}$ of $C^{k}\left(X ; \mathbb{Z}^{n}\right)$ which will be denoted by $C_{L^{\infty}}^{k}\left(X ; \mathbb{Z}^{n}\right)$.

Lemma 8.2. Suppose $k>0, X$ is a $C W$-complex with finitely many ( $k-$ 1)-cells, $\tilde{X} \stackrel{p}{\rightarrow} X$ is the universal covering, and $\alpha \in B^{k}\left(X ; \mathbb{Z}^{n}\right)$ is a $k$ coboundary. Then $p^{*} \alpha \in \operatorname{Im}\left(C_{L^{\infty}}^{k-1}\left(\tilde{X} ; \mathbb{Z}^{n}\right) \stackrel{\delta}{\rightarrow} C^{k}\left(\tilde{X} ; \mathbb{Z}^{n}\right)\right)$.

\footnotetext{
${ }^{8}$ Under appropriate finiteness conditions $C_{L^{\infty}}^{*}\left(X ; \mathbb{Z}^{n}\right)$ will be a subcomplex of $C^{*}\left(X ; \mathbb{Z}^{n}\right)$ and the $L^{\infty}$ cohomology $H_{L^{\infty}}^{*}\left(X ; \mathbb{Z}^{n}\right)$ will be well-defined.
} 
Proof. If $\theta \in C^{k-1}\left(X ; \mathbb{Z}^{n}\right)$ and $\alpha=\delta \theta$ then $p^{*} \alpha=p^{*} \delta \theta=\delta p^{*} \theta$ and $p^{*} \theta \in$ $C_{L^{\infty}}^{k-1}\left(\tilde{X} ; \mathbb{Z}^{n}\right)$ since $X$ has a finitely many $(k-1)$ cells.

Let $X$ be a CW complex with finitely many $(k-1)$-cells. By the Lemma, the subgroup

$$
Z_{s p}^{k}\left(X ; \mathbb{Z}^{n}\right):=\left\{\alpha \in Z^{k}\left(X ; \mathbb{Z}^{n}\right) \mid p^{*} \alpha \in \operatorname{Im}\left(C_{L^{\infty}}^{k-1}\left(\tilde{X} ; \mathbb{Z}^{n}\right) \stackrel{\delta}{\rightarrow} C^{k}\left(\tilde{X} ; \mathbb{Z}^{n}\right)\right)\right\}
$$

descends to a subgroup $H_{s p}^{k}(X ; \mathbb{Z})$ of $H^{k}\left(X ; \mathbb{Z}^{n}\right)$; we will refer elements of $H_{s p}^{k}\left(X ; \mathbb{Z}^{n}\right)$ as special cohomology classes ${ }^{9}$. If $X \stackrel{f}{\rightarrow} X^{\prime}$ is a continuous map from $X$ to another CW complex with finitely many $(k-1)$-cells, we can homotope $f$ to a cellular map, so we have an induced homomorphism

$$
H_{s p}^{k}\left(X^{\prime} ; \mathbb{Z}^{n}\right) \stackrel{f^{*}}{\rightarrow} H_{s p}^{k}\left(X ; \mathbb{Z}^{n}\right) .
$$

When $G$ is a finitely generated group, the special cohomology group $H_{s p}^{2}\left(G ; \mathbb{Z}^{n}\right)$ of $G$ is defined as follows: pick a $K(G, 1)$ with finite 1-skeleton; the special cohomology group $H_{s p}^{2}\left(X ; \mathbb{Z}^{n}\right) \subset H^{2}\left(X ; \mathbb{Z}^{n}\right) \simeq H^{2}\left(G ; \mathbb{Z}^{n}\right)$ defines a subgroup of $H^{2}\left(G ; \mathbb{Z}^{n}\right)$ which is independent of the choice of $X$.

Proposition 8.3. Let

$$
1 \rightarrow \mathbb{Z}^{n} \stackrel{i}{\rightarrow} G \stackrel{p}{\rightarrow} Q \rightarrow 1
$$

be a central extension of finitely generated groups. Then the following are equivalent:

1. The extension is quasi-isometrically trivial, i.e. there is a quasiisometry $G \stackrel{f}{\longrightarrow} \mathbb{Z}^{n} \times Q$ so that the diagram

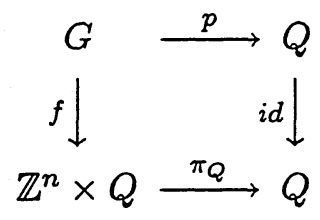

commutes up to bounded error.

2. There is a Lipschitz section $s: Q \rightarrow G$ of $p$.

\footnotetext{
9It would be more descriptive to say that these classes "pullback to $d$ (bounded)"; but we chose "special" for brevity.
} 
3. The cohomology class $\alpha \in H^{2}\left(G ; \mathbb{Z}^{n}\right)$ associated with the extension (8.4) is a special cohomology class, i.e. if $K$ is a $K(G, 1)$ with finite 1 -skeleton and $c \in Z^{2}\left(K ; \mathbb{Z}^{n}\right)$ represents $\alpha$, then $p^{*} c \in$ $\operatorname{Im}\left(H_{L^{\infty}}^{1}\left(\tilde{K} ; \mathbb{Z}^{n}\right) \stackrel{\delta}{\rightarrow} H^{1}\left(\tilde{K} ; \mathbb{Z}^{n}\right)\right)$.

Proof. $(1 \Longrightarrow 2$ ). Suppose $f$ makes diagram (8.5) commute up to bounded error, and let $f^{-1}$ be a quasi-inverse ${ }^{10}$ for $f$. Define $s_{0}: Q \rightarrow G$ to be the composition $Q \rightarrow\{e\} \times Q \rightarrow \mathbb{Z}^{n} \times Q \stackrel{f^{-1}}{\rightarrow} G$. The approximate commutativity of (8.5) implies that $d\left(p \circ s_{0}, i d_{Q}\right)<\infty$. Define a section $s: Q \rightarrow G$ of $p$ by letting $s(q)$ be a point in $p^{-1}(q)$ closest to $s_{0}(q)$, for all $q \in Q$. By Lemma 8.6 below, we have $d\left(s, s_{0}\right)<\infty$, and so $s$ is Lipschitz since $s_{0}$ is Lipschitz and $d\left(q_{1}, q_{2}\right) \geq 1$ for distinct elements $q_{1}, q_{2} \in Q$.

Lemma 8.6. If $H \triangleleft G$ are finitely generated groups, we define a distance function $d_{G / H}$ on $G / H$ by letting $d_{G / H}\left(g_{1} H, g_{2} H\right)$ be the distance between the subsets $g_{1} H, g_{2} H$ of $G$ with respect to a fixed word metric on $G$. Then the coset distance metric on $G / H$ is equivalent ${ }^{11}$ to any word metric on $G / H$.

Proof. Let $\Sigma \subset G$ be a symmetric finite generating set, and let $\bar{\Sigma} \subset G / H$ be the image of $\Sigma$ under $G \rightarrow G / H$. Then there is a canonical 1-Lipschitz map between the Cayley graphs $\operatorname{Cay}(G, \Sigma)$ and $\operatorname{Cay}(G / H, \bar{\Sigma})$. Paths in $\operatorname{Cay}(G / H, \bar{\Sigma})$ can be lifted to paths in $\operatorname{Cay}(G, \Sigma)$ of the same length which join the corresponding cosets of $H$.

$(2 \Longrightarrow 1)$. If $s: Q \rightarrow G$ is a Lipschitz section of $p$, we may define a map $\pi_{\mathbb{Z}^{n}}: G \rightarrow \mathbb{Z}^{n}$ by the formula $\pi_{\mathbb{Z}^{n}}(g) s(p(g))=g$, i.e. $\pi_{\mathbb{Z}^{n}}$ is the unique map $G \rightarrow \mathbb{Z}^{n}$ which sends $s(Q)$ to $e \in \mathbb{Z}^{n}$, and which is equivariant with respect to translation by elements of $\mathbb{Z}^{n}$.

Lemma 8.7. $\pi_{\mathbb{Z}^{n}}$ is Lipschitz.

Proof. Note that if $g_{1}, g_{2} \in G, h \in \mathbb{Z}^{n}$, and $g_{2}=g_{1} h$, then $\pi_{\mathbb{Z}^{n}}\left(g_{2}\right)=$ $\pi_{\mathbb{Z}^{n}}\left(g_{1}\right) h$, so $d_{\mathbb{Z}^{n}}\left(\pi_{\mathbb{Z}^{n}}\left(g_{1}\right), \pi_{\mathbb{Z}^{n}}\left(g_{2}\right)\right)=d_{\mathbb{Z}^{n}}(e, h)$. The properness of the distance function $d_{\mathbb{Z}^{n}}(\cdot, e)$ implies that there is a function $\delta: \mathbb{N} \rightarrow \mathbb{N}$ so that

${ }^{10} d\left(f^{-1} \circ f, i d_{G}\right)$ and $d\left(f \circ f^{-1}, i d_{\mathbb{Z}^{n} \times Q}\right)$ are both finite.

${ }^{11}$ The two metrics have uniformly bounded ratio. 
for all $h \in \mathbb{Z}^{n}$,

$$
d_{\mathbb{Z}^{n}}(h, e) \leq \delta\left(d_{G}(h, e)\right) .
$$

To prove Lemma 8.7, it suffices to find an $L$ such that

$$
d_{\mathbb{Z}^{n}}\left(\pi_{\mathbb{Z}^{n}}\left(g_{1}\right), \pi_{\mathbb{Z}^{n}}\left(g_{2}\right)\right) \leq L
$$

whenever $d_{G}\left(g_{1}, g_{2}\right)=1$. Consider the unique $g_{3} \in g_{1} \mathbb{Z}^{n}$ which satisfies $\pi_{\mathbb{Z}^{n}}\left(g_{3}\right)=\pi_{\mathbb{Z}^{n}}\left(g_{2}\right)$, i.e. $g_{3} \in g_{1} \mathbb{Z}^{n} \cap\left(\pi_{\mathbb{Z}^{n}}\left(g_{2}\right) s(Q)\right)$. Then $d_{G}\left(g_{3}, g_{2}\right) \leq C$ for some constant $C$ because the composition $s \circ p$ is Lipschitz. Applying triangle inequalities and (8.8), we get

$$
\begin{aligned}
d_{\mathbb{Z}^{n}}\left(\pi_{\mathbb{Z}^{n}}\left(g_{1}\right), \pi_{\mathbb{Z}^{n}}\left(g_{2}\right)\right) & =d_{\mathbb{Z}^{n}}\left(\pi_{\mathbb{Z}^{n}}\left(g_{1}\right), \pi_{\mathbb{Z}^{n}}\left(g_{3}\right)\right) \\
& \leq \delta\left(d_{G}\left(g_{1}, g_{3}\right)\right) \leq \delta(1+C) .
\end{aligned}
$$

To finish the proof that $(2 \Longrightarrow 1)$, note that we have a bijection $\hat{f}$ : $\mathbb{Z}^{n} \times Q \rightarrow G$ given by $\hat{f}(h, q)=h s(q)$. $\hat{f}$ is clearly $\operatorname{Lip}(s)$-Lipschitz in the $Q$ direction. That $\hat{f}$ is Lipschitz in the $\mathbb{Z}^{n}$ direction follows from the fact that $\mathbb{Z}^{n}$ is a central subgroup of $G$ :

$$
\begin{aligned}
d_{G}\left(\hat{f}\left(h_{1}, q\right), \hat{f}\left(h_{2}, q\right)\right) & =d_{G}\left(h_{1} s(q), h_{2} s(q)\right) \\
& =d_{G}\left(h_{1} h_{2}^{-1}, e\right) \leq d_{\mathbb{Z}^{n}}\left(h_{1} h_{2}^{-1}, e\right)=d_{\mathbb{Z}^{n}}\left(h_{1}, h_{2}\right) .
\end{aligned}
$$

Letting $f=\hat{f}^{-1}$, we see that $f=\left(\pi_{\mathbb{Z}^{n}}, p\right)$ is a biLipschitz bijection.

$(2 \Longleftrightarrow 3)$. This follows from the obstruction theoretic interpretation of the characteristic class of the extension. Let $K$ be a CW complex with finite 1skeleton and one vertex, and which is an Eilenberg-Maclane space for $Q$. Let $P \rightarrow K$ be a principal $T^{n}$-bundle with characteristic class $[\alpha] \in H^{2}\left(K ; \mathbb{Z}^{n}\right)$, so that the exact homotopy sequence $\pi_{1}\left(T^{n}\right) \rightarrow \pi_{1}(P) \rightarrow \pi_{1}(K)$ for the fibration $P \rightarrow K$ is isomorphic to (8.4). Let $\sigma: \operatorname{Skel}_{1}(K) \rightarrow P$ be a section of $P$ over the 1-skeleton of $K$. In the fiber over the point $S k e l_{0}(K)$, choose a bouquet of $n$ circles with vertex at $\sigma\left(\operatorname{Skel}_{0}(K)\right)$, which gives a standard basis for the fundamental group of the fiber. Let $M \subset P$ be the 1-complex consisting of the union of this bouquet of circles with the bouquet $\sigma\left(\operatorname{Skel}_{1}(K)\right) \subset P$.

Let $\hat{P} \rightarrow \tilde{K}$ be the pullback of the bundle $P \rightarrow K$ under the covering projection $\tilde{K} \rightarrow K$, let $\hat{\sigma}: S k e l_{1}(\tilde{K}) \rightarrow \hat{P}$ be the pullback of $\sigma$, and let 
$\hat{M} \subset \hat{P}$ be the inverse image of $M$ under the covering $\hat{P} \rightarrow P$. Finally, let $\tilde{P} \rightarrow \hat{P}$ be the universal covering, and let $\tilde{M} \subset \tilde{P}$ be the inverse image of $\hat{M}$ under $\tilde{P} \rightarrow \hat{P}$. Note that if we put path metrics on $\operatorname{Skel}_{1}(\tilde{K})$ and $\tilde{M}$, then the projection map $\operatorname{Skel}_{0}(\tilde{M}) \rightarrow \operatorname{Skel}_{0}(\tilde{K})$ is naturally biLipschitz equivalent to $G \stackrel{p}{\rightarrow} Q$.

Now suppose 3 holds, and that $\alpha \in C_{L^{\infty}}^{2}\left(K ; \mathbb{Z}^{n}\right) \subset C^{2}\left(K ; \mathbb{Z}^{n}\right)$. We may assume that our section $\sigma: \operatorname{Skel}_{1}(K) \rightarrow P$ was chosen so that the associated cellular obstruction cocycle is $\alpha$. Then $\hat{\alpha}$, the image of $\alpha$ under the pullback $C_{L^{\infty}}^{2}\left(K ; \mathbb{Z}^{n}\right) \rightarrow C_{L^{\infty}}^{2}\left(\tilde{K} ; \mathbb{Z}^{n}\right)$, is the obstruction cocycle for $\hat{\sigma}: \operatorname{Skel}_{1}(\tilde{K}) \rightarrow \hat{P}$. By assumption, $\hat{\alpha}=\delta \theta$ for some $\theta \in C_{L^{\infty}}^{1}\left(\tilde{K} ; \mathbb{Z}^{n}\right)$. Hence we may modify $\hat{\sigma}$ using $\theta$ to get a new section $\hat{\sigma}_{1}: \operatorname{Skel}_{1}(\tilde{K}) \rightarrow \hat{P}$ with trivial obstruction cocycle. In particular, if $\tilde{P} \rightarrow \hat{P}$ is the universal covering map, then $\hat{\sigma}_{1}$ lifts to a section $\tilde{\sigma}: \operatorname{Skel}_{1}(\tilde{K}) \rightarrow \tilde{P}$ of the $\mathbb{R}$-bundle $\tilde{P} \rightarrow \tilde{K}$. The fact that $\theta$ is an $L^{\infty}$-cochain implies that $\tilde{\sigma}$ restricts to a 1-Lipschitz map from $\operatorname{Skel}_{0}(\tilde{K})$ to $\operatorname{Skel}_{0}(\tilde{M})$. Since the projection $\operatorname{Skel}_{0}(\tilde{M}) \rightarrow \operatorname{Skel}_{0}(\tilde{K})$ is biLipschitz equivalent to $G \rightarrow Q$, we get a Lipschitz section of $p$, so 2 holds.

Conversely, suppose 2 holds. Then we get a Lipschitz section $\tau$ : $\operatorname{Skel}_{0}(\tilde{K}) \rightarrow \operatorname{Skel}_{0}(\tilde{M})$ of the projection $\operatorname{Skel}_{0}(\tilde{M}) \rightarrow S_{k e l_{0}}(\tilde{K})$. We may extend $\tau$ to a section $\tilde{\sigma}: S k e l_{1}(\tilde{K}) \rightarrow \tilde{P}$, and let $\hat{\sigma}_{1}: S k e l_{1}(\tilde{K}) \rightarrow \hat{P}$ be the composition of $\tilde{\sigma}$ with $\tilde{P} \rightarrow \hat{P}$. Notice that $\hat{\sigma}_{1}$ has trivial obstruction cocycle since it lifts to $\tilde{\sigma}$.

Lemma 8.9. $\hat{\sigma}_{1}$ is obtained from $\hat{\sigma}$ by applying a bounded cochain $\theta \in$ $C_{L^{\infty}}^{1}\left(\tilde{K} ; \mathbb{Z}^{n}\right)$.

Proof. If $e$ is a closed 1-cell in $\operatorname{Skel}_{1}(\tilde{K})$, we want to show that the fixed endpoint homotopy classes of the two sections $\left.\hat{\sigma}\right|_{e}: e \rightarrow \hat{P}$ and $\left.\hat{\sigma}_{1}\right|_{e}: e \rightarrow \hat{P}$ (as maps into the inverse image of $e$ in $\hat{P}$ ) agree up to bounded error. If $\gamma:[0,1] \rightarrow e$ is a characteristic map for $e$, lift the path $\hat{\sigma} \circ \gamma:[0,1] \rightarrow \hat{M} \subset \hat{P}$ to a path $\tilde{\gamma}:[0,1] \rightarrow \tilde{M} \subset \tilde{P}$ starting at $\tilde{\sigma} \circ \gamma(0)$. Then

$$
\begin{aligned}
d_{\tilde{M}}(\tilde{\gamma}(1), \tilde{\sigma} \circ \gamma(1)) & \leq d_{\tilde{M}}(\tilde{\gamma}(1), \tilde{\gamma}(0))+d_{\tilde{M}}(\tilde{\gamma}(0), \tilde{\sigma} \circ \gamma(1)) \\
& =1+d_{\tilde{M}}(\tau(\gamma(0)), \tau(\gamma(1))) \\
& \leq 1+L_{\tau}
\end{aligned}
$$

where $L_{\tau}$ is the Lipschitz constant of $\tau$. But then $\tilde{\gamma}(1)=(\tilde{\sigma} \circ \gamma(1)) h$ for some $h \in \mathbb{Z}^{n}$, and we can bound $d_{\mathbb{Z}^{n}}(h, e)$ by a constant $C$ depending on $L_{\tau}$, cf. (8.8). In other words, the fixed endpoint homotopy classes of $\left.\hat{\sigma}\right|_{e}$ and 
$\left.\hat{\sigma}_{1}\right|_{e}$ (as maps from $e$ to the inverse image of $e$ in $\hat{P}$ ) differ by some $h \in \mathbb{Z}^{n}$ where $\|h\|_{\mathbb{Z}^{n}}<C$.

If $\alpha \in C^{2}\left(K ; \mathbb{Z}^{n}\right)$ is the obstruction cocycle for the section $\sigma$, then the pullback of $\alpha$ to $\hat{K}$ is the obstruction for $\hat{\sigma}$. As the obstruction for $\hat{\sigma}_{1}$ is 0 , Lemma 8.9 gives

$$
0=\alpha+\delta \theta
$$

for $\theta \in C_{L^{\infty}}^{1}\left(\tilde{K} ; \mathbb{Z}^{n}\right)$, so 3 holds. This completes the proof of Proposition 8.3.

\section{References.}

[CJ94] A. Casson and D. Jungreis, Convergence groups and Seifert fibered 3manifolds, Inv. Math. 118 (1994), 441-456.

[Chow96] R. Chow, Groups quasi-isometric to complex hyperbolic space, Trans. AMS, 348 (1996), 1757-1769.

[Esk96] A. Eskin, Quasi-isometric rigidity of nonuniform lattices in higher rank symmetric spaces, J. AMS, 11 (1998), 321-361.

[Ga92] D. Gabai, Convergence groups are Fuchsian groups, Ann. Math. 136 (1992), 447-510.

[Ger92] S. Gersten, Bounded cocycles and combings of groups, Int. J. Alg. Comp., 2 (1992), 307-326.

[Ger91] S. Gersten, H. Short, Rational subgroups of biautomatic groups, Ann. of Math. 134 (1991), 125-158.

[GDH90] E. Ghys, P. de la Harpe, Sur les groupes hyperboliques d'apres Mikhael Gromov, Progress in Mathematics, 83, Birkhäuser.

[Gro93] M. Gromov, Asymptotic invariants for infinite groups, in: Geometric group theory, London Math. Soc. lecture note series 182, 1993.

[Gro81a] M. Gromov, Hyperbolic manifolds, groups, and actions, Riemann surfaces and related topics: Proceedings of the 1978 Stony Brook Conference, 183213, Ann. of Math. Stud. 97.

[Gro81b] M. Gromov, Groups of polynomial growth and expanding maps, Publ. IHES, 53 (1981), 53-73.

[Gro87] M. Gromov, Hyperbolic groups, 75-263, In: Essays in group theory, MSRI Publ. 8, Springer, 1987. 
[Hin90] A. Hinkkanen, The structure of certain quasi-symmetric groups, Mem. Amer. Math. Soc. 83 (1990), 1-83.

[KaLe96] M. Kapovich and B. Leeb, Quasi-isometries preserve the geometric decomposition of Haken manifolds, Invent. Math. 128 (1997), 393-416.

[KlLe97a] B. Kleiner and B. Leeb, Rigidity of quasi-isometries for symmetric spaces and Euclidean buildings, C. R. Acad. Sci. Paris, 324 (1997), 639-643.

[KILe97b] B. Kleiner and B. Leeb, Rigidity of quasi-isometries for symmetric spaces and Euclidean buildings, Publ. IHES, vol. 86 (1997), 115-197.

[Pan83] P. Pansu, Croissance des boules et des géodésiques fermées dans les nilvariétés, Erg. Thy. Dyn. Sys. 3 (1983), 415-445.

[Pan89] P. Pansu, Métriques de Carnot-Carathéodory et quasiisométries des espaces symetriques de rang un, Ann. of Math. 129 (1989), 1-60.

[Rag84] M.S. Ragunathan, Torsion in cocompact lattices in coverings of $\operatorname{Spin}(2, n)$, Math. Ann. 266 (1984), 403-419.

[ReNe97] W.D. Neumann, L. Reeves, Central extensions of word hyperbolic groups, Ann. of Math. (2) 145 (1997), 183-192.

[Rie93] E. Rieffel, Groups coarse quasi-isometric to $\mathbb{H}^{2} \times \mathbb{R}, \mathrm{PhD}$ Thesis, UCLA, 1993.

[Sch95] R. Schwartz, The quasi-isometry classification of rank one lattices, Publ. of IHES, vol. 82 (1995), 133-168.

[Tuk86] P. Tukia, On quasiconformal groups, J. Analyse Math. 46 (1986), 318-346.

[Tuk88] P. Tukia, Homeomorphic conjugates of Fuchsian groups, J. Reine Angew. Math. 391 (1988), 1-54.

[TuVa82] P. Tukia, J. Väisälä, Quasiconformal extension from dimension $n$ to $n+1$, Ann. Math., 115 (1982), 331-348.

UNIVERSITY OF MICHIGAN

ANN ARBOR, MI 48109-1109

AND

UNIVERSITÄT OF TÜBINGEN

E-mail addresses: bkleiner@math.Isa.umich.edu

leeb@Mathematik.Uni-Mainz.DE 
RECEIVED November 12, 1998. 